$\boldsymbol{L E X}$. Revista de Investigaciones en Ciencias Jurídicas ocubre-diciembre-2018

Volumen 1, Número 2

pp.29-36

\title{
Valoración y limitaciones de los medios de pruebas electrónicos en el proceso laboral venezolano
}

\section{Valuation and limitations of the means of evidence electronics in the venezuelan labor process}

\section{Avaliação e limitações dos meios de evidência eletrônica no processo de trabalho venezuelano}

\author{
Belkis Zoraida Gainza Lovera \\ belkisgainza@hotmail.es \\ Poder Judicial Venezolano, Venezuela
}

Articulo recibido en febrero 2018; arbitrado en marzo 2018; aprobado en abril 2018 y publicado junio de 2018

RESUMEN

La valoración probatoria y las limitaciones de los diversos medios de pruebas electrónicos en el proceso laboral venezolano, para garantizar el derecho a la defensa y al debido proceso previsto en la norma constitucional. Se diferencia el documento electrónico del documento efectuado en soporte de papel; precisando la forma adecuada de promover, evacuar y valorar las pruebas que consten en instrumentos electrónicos. Fue un documento de tipo documental monográfico, utilizando un método sistemático a través de la deducción-inducción. Concluyendo que la noción de documento, situado ya dentro del ámbito jurídico, es aquella que entiende por tal; aquellos objetos que tengan una función probatoria con la sola limitación de que dichos objetos sean, por su índole, susceptibles de ser llevados ante la presencia judicial y la esencia del documento electrónico se encuentra en su almacenamiento a través de medios informáticos.

Palabras clave: Prueba; Valoración; documento electrónico; mensajes electrónicos
ABSTRACT

The probative assessment and limitations of the various means of electronic evidence in the Venezuelan labor process, to guarantee the right to defense and due process provided for in the constitutional norm. The electronic document differs from the document made on paper support; specifying the appropriate way to promote, evacuate and evaluate the evidence contained in electronic instruments. It was a document of monographic documentary type, using a systematic method through deduction-induction. Concluding that the notion of a document, which is already within the legal scope, is that which it understands as such; those objects that have a probative function with the only limitation that such objects are, by their nature, susceptible to being brought before the judicial presence and the essence of the electronic document is in its storage through computerized means.

Key words: Test; Assessment; electronic document; emails 
RESUMO

Avaliação de provas e limitações dos vários meios de provas eletrônicas no processo de trabalho da Venezuela, para garantir o direito de defesa e do devido processo sob a lei constitucional. 0 documento eletrônico difere do documento feito em suporte de papel; especificando a maneira apropriada de promover, evacuar e avaliar as evidências contidas em instrumentos eletrônicos. Foi um documento do tipo documentário monográfico, utilizando um método sistemático através da dedução-

\section{INTRODUCCIÓN}

Medios de prueba electrónicos en el proceso laboral venezolano, tiene relevancia, con el fin de disponer de un estudio que permita dar herramientas de la forma de promover los medios electrónicos y el sistema de valoración aplicado en cada caso.

Por otra parte, su estudio también está vinculado al área procesal, intrínsecamente relacionado con el Proceso Civil vigente, lo que permite analizar la valoración de uno y otro proceso, en cuanto a sus semejanzas y diferencia.

Es bien sabido, que la era de las telecomunicaciones está en su máximo desarrollo; y Venezuela no ha escapado a esto, ya que, en tan solo dos décadas se ha transformado la sociedad venezolana, hoy, un importante número de ciudadanos incluyendo la población infantil, hacen uso de celulares y mantienen a diario algún tipo de contacto vía Internet.

Hoy en día hay, una significativa importancia, de las telecomunicaciones debido a que aporta información sobre los diversos medios de pruebas electrónicos; lo que constituye un beneficio económico, ya que permite reducir los tiempos y costos originados, en la resolución de los conflictos en los que se deban valorar medios de pruebas electrónicos no comunes en el ámbito procesal y pocos regulados en nuestro ordenamiento jurídico. indução. Concluindo que a noção de documento, que já se encontra no âmbito jurídico, é aquela que entende como tal; os objetos que têm uma função à prova com a única limitação que disse objetos são, pela sua natureza, capaz de ser trazido à presença justiça e essência do documento eletrônico está no armazenamento por meio de computador.

Palavras chave: Teste; Avaliação; documento eletrônico; mensagens eletrônicas

Es relevante, la incidencia de la tecnología, concretamente en lo relativo al campo de las telecomunicaciones a través de Internet y telefonía celular, como elementos determinantes en la sociedad moderna, lo cual trae como consecuencia inevitable que cada vez más se encuentren presentes tales elementos en los procesos judiciales $\mathrm{y}$ administrativos.

De ahí que, parece irreversible que estos nuevos instrumentos sustituirán a los acostumbrados, ello se refleja en la cantidad de documentos que se producen elaborados electrónicamente. Por esa razón, es importante preguntarse si se dispone de la normativa e infraestructura material y humana que permita la regulación y valoración jurídica apropiada. De ser positiva la respuesta a ésta interrogante debe tenerse en cuenta que la normativa debe ser futurista previendo que, es una realidad que los cambios tecnológicos avanzan de manera sorprendente y acelerada; caso contrario sería inminente tomar las medidas necesarias para estrechar las diferencias existente entre la realidad social y el derecho.

No puede dejar de mencionarse, lo relevante del tema específicamente para los abogados litigantes que a menudo se le presentará la necesidad de hacer valer uno de estos medios de prueba, y aún más para el juez que es quien debe valorarlos, y en términos más amplios la importancia que 
reviste para la sociedad que requiere seguridad jurídica y en lo posible unidad de criterio judicial con respecto a estos medios que, a pesar de su cotidianidad aun no gozan de un respaldo jurídico consistente.

Por otra parte, Devis-Echandía (1981), plantea que:

En el ámbito jurídico, se entiende por documento como una cosa representativa, o sea capaz de representar un hecho. Pero desde un punto de vista un poco más restringido es posible concebir el documento como toda cosa que sea producto de un acto humano, perceptible con los sentidos de la vista y el tacto, que sirve de prueba histórica indirecta y representativa de un hecho cualquiera. (p. 486).

En un plano más avanzado de concreción de la noción y transitando desde el campo genérico del derecho instrumental al de la prueba civil puede entenderse por tal, todo escrito en soporte papel. Así explica Jijena (1998) que un documento en sentido estricto es, "todo recipiente en el cual se vierten por escrito manifestaciones $o$ declaraciones. Estrictamente entendidos, los documentos son escritos o escrituras". (p.1497).

Por lo tanto, es innegable la necesidad de determinar con certeza como es la manera adecuada de promover, evacuar, controlar y valorar los mismos; cuales son los requisitos que deben reunir estos documentos para que puedan hacerse valer en juicio, entre otras muchas interrogantes que se plantean al hacerse necesario probar por estas vías.

\section{MÉTODO}

El estudio fue descriptivo, dado que a través del análisis con sentido crítico y temático de los diferentes textos legales, jurisprudenciales y doctrinales, se logran los objetivos planteados.

Asimismo, Tamayo y Tamayo (1999), refiere que:

...mediante este tipo de investigación descriptiva, que utiliza el método de análisis, se logra caracterizar un objeto de estudio o una situación concreta, señalar sus características y propiedades. Combinada con ciertos criterios de clasificación sirve para ordenar, agrupar o sistematizar los objetos involucrados en el trabajo indagatorio. Al igual que la investigación que se ha descrito anteriormente, puede servir de base para investigaciones que requieran un mayor nivel de profundidad (p. 72).

Lo anterior configura, una investigación analítica y de desarrollo conceptual, con apoyo de una amplia revisión bibliográfica. Siendo un diseño de acuerdo a las características y objetivos propuestos.

En el mismo orden de ideas para Arias (2006) una investigación documental es un proceso basado en la búsqueda, recuperación, análisis, crítica e investigación de los datos secundarios, es decir, los datos obtenidos y registrados por otros investigadores en fuentes documentales. (p. 27)

Por lo tanto, el estudio estuvo enmarcado bajo la modalidad de investigación documental, dado que se realizó la búsqueda y análisis de la literatura que aportaron información que dio paso a la comprensión de la problemática del estudio, así como el análisis y valoración de los medios de prueba electrónicos en el proceso laboral venezolano, desde la perspectiva formal con base en el fundamento teórico y a la abstracción del tema objeto de estudio.

Al respecto Tamayo y Tamayo (2005) señala que: "Un diseño bibliográfico es cuando recurrimos a la utilización de datos secundarios, es decir, aquellos que han sido obtenidos por otros y nos llegan elaborados y procesados de acuerdo con los fines de quienes inicialmente los elaboran, procesan y manejan". (p 109)

\section{RESULTADOS}

Diferencia entre el documento electrónico y el documento efectuado en soporte de papel

Un documento es un testimonio material de un hecho o acto realizado en el ejercicio de 
sus funciones por instituciones o personas físicas, jurídicas, públicas o privadas, registrado en una unidad de información cualquier tipo de soporte (papel, cintas, discos magnéticos, fotografías, entre otros) en lenguaje natural o convencional. Es el testimonio de una actividad humana fijada en un soporte.

Tradicionalmente, el medio de un documento era el papel y la información era ingresada a mano, utilizando tinta (esto es lo que se denomina hacer un manuscrito) o por un proceso mecánico (mediante una máquina de escribir, o utilizando una impresora láser).

En el ámbito jurídico, dentro de los exponentes clásicos de la noción amplia de documento encontramos a Carnelutti (1982), quien entiende por documento; «una cosa representativa, o sea capaz de representar un hecho» (p.156), o como explica el autor citado, la representación es la imagen de la realidad, la que se presenta al intelecto a través de los sentidos; y, en consecuencia, el documento es una cosa que sirve para representar a otra. Continúa señalando Carnelutti, que la representación de un hecho, y no la manifestación del pensamiento es la nota esencial al concepto de documento.

Quienes sostenían la acepción amplia del concepto -teoría de la representación-, admitían al documento electrónico dentro de la categoría documental, mientras quienes abogaban por la 'teoría del escrito', excluían, como lógica consecuencia de la concepción doctrinal sostenida, al documento electrónico de la categoría documental, al entender ésta exclusivamente como el escrito contenido en soporte papel.

El documento es definido en la Ley Especial Contra Los Delitos Informáticos, (2001) en su artículo 2 literal e, como un "registro incorporado en un sistema en forma de escrito, video, audio o cualquier otro medio, que contiene data o información acerca de un hecho o acto capaces de causar efectos jurídicos" (p. 4)

\section{Documento electrónico}

En cuanto, al documento electrónico o informático, Barriuso, (1998), señala que el documento electrónico lo constituyen "las declaraciones de voluntad con efectos de creación, modificación o extinción de derechos y obligaciones, por medio de la electrónica, informática y telemática (p. 319)

Los documentos electrónicos son una especie dentro del género de los documentos, y los mismos han nacido del uso cada vez más generalizado de las tecnologías de la información, así como de la masificación de las telecomunicaciones, y con ellos el comercio electrónico, obligando al derecho a desarrollar regulaciones que permitan el uso de dichas tecnologías.

Para Davara, (1998) el documento en soporte electrónico, informático y telemático es un documento con las mismas características, en principio y en cuanto a su validez jurídica, que cualquier otro de los que tradicionalmente se aceptan en soporte de papel. (p. 97)

Formas de promover y evacuar las pruebas que consten en instrumentos electrónicos, delimitando el ejercicio del control de la prueba y sus limitaciones

El documento electrónico no se encuentra regulado en forma expresa en la mayoría de las normas procesales, lo cual plantea una triple problemática al respecto: en primer lugar, su admisión en el proceso, en segundo lugar, la forma de incorporarlo a éste y por último, el valor probatorio atribuido en relación con los demás medios de prueba.

\section{Admisión}

En España, antes de la promulgación de la Ley de Enjuiciamiento Civil (LEC) de 2000 que establece el principio de libertad en los medios de prueba, la admisión del documento electrónico ya había sido reconocida por la jurisprudencia del Tribunal Supremo Español (TSE).

La sentencia de la Sala 2a de lo Penal de 5 de febrero de 1988, que trata sobre el valor 
probatorio de una grabación telefónica, dejó sentado que los medios de prueba establecidos en las leyes de procedimiento no tienen el carácter exhaustivos, por lo que las innovaciones tecnológicas deben sumarse al acervo jurídico procesal. En la misma sentencia, el TSE al referirse a los modernos medios de prueba -cine, video y cintas magnéticas- indica que tanto éstos como los ordenadores electrónicos constituyen una expresión de la realidad que el derecho no puede desconocer, agregando, dichos medios técnicos pueden subsumirse en el concepto, amplio desde luego, de documento".

Siguiendo los criterios jurisprudenciales, la LEC española de 2000, reconoce como medio de prueba los soportes informáticos, a tal efecto, el artículo 299 establece: "También se admitirán, conforme a lo dispuesto en esta Ley, los medios de reproducción de la palabra, el sonido y la imagen, así como los instrumentos que permiten archivar $y$ conocer o reproducir palabras, datos. (p 136)

El artículo se refiere a dos situaciones claramente diferenciadas, en primer lugar, alude a los medios de reproducción de la palabra, sonido e imagen, donde se incluyen los medios de reproducción audiovisual y en segundo lugar a los instrumentos que permiten archivar, conocer $y$ reproducir datos, identificados por la doctrina española como soportes informáticos.

En Venezuela, el artículo 395 del Código de Procedimiento Civil (CPC), (1990) consagra el principio de libertad de los medios de prueba al indicar que: "Son medios de pruebas admisibles en juicio aquellos que determina el Código Civil, el presente Código y otras leyes de la República" (p. 78), esta libertad probatoria la encontramos también en el propio texto de la Constitución de la República Bolivariana de Venezuela, (1999) a tenor del artículo 49, el cual señala que "toda persona tiene derecho a disponer de los medios adecuados para ejercer su defensa". (p. 41).

La Ley Orgánica Procesal del Trabajo (2002) establece en su artículo 70:
Son medios de pruebas admisibles en juicio aquellos que determine la presente ley, el Código de Procedimiento Civil, el Código Civil, y otras leyes de la República; quedan excluidas las pruebas de posiciones juradas juramento decisorio Las partes pueden también, valerse de cualquier otro medio de prueba no prohibido expresamente por la ley $y$ que consideren conducente a la demostración de sus pretensiones. Estos medios se promoverán y evacuaran, de la forma preceptuada en la presente Ley, en lo no previsto en esta se aplicarán, por analogía, las disposiciones relativas a los medios de pruebas semejantes contemplados en el Código de Procedimiento Civil, el Código Civil, o en su defecto, en la forma que señale el Juez del Trabajo. (p. 63).

La Ley Sobre Mensajes de Datos y Firmas Electrónicas (LMDFE) (2001), de Venezuela consagra la eficacia probatoria del documento electrónico al establecer en su artículo 4:

Los Mensajes de Datos tendrán la misma eficacia probatoria que la ley otorga a los documentos escritos, sin perjuicio de lo establecido en la primera parte del artículo 6 de este Decreto-Ley. Su promoción, control, contradicción y evacuación como medio de prueba, se realizará conforme a lo previsto para las pruebas libres en el Código de Procedimiento Civil. (p. 6).

Respecto a las pruebas libres, el CPC establece la aplicación de la analogía en relación con los medios semejantes contemplados en el Código Civil (CC), por tanto los mensajes de datos deben considerarse admisibles en el concepto de documentos, aplicándose las normas de valoración establecidas en la Ley para estos casos.

Según manifiesta Rico, (2005) en el derecho Italiano, el artículo 5 del decreto del presidente de la República (513/97), 
establece "la admisibilidad del documento electrónico como medio probatorio siempre que esté signado con la firma digital en las condiciones exigidas por el artículo 10 del reglamento, otorgándole eficacia probatoria de escritura privada" (p. 105)

En Venezuela, la Ley Orgánica Procesal del Trabajo, (2002) establece en su artículo 75 para la admisión de pruebas lo siguiente:

Dentro de los cinco días hábiles siguientes al recibo del expediente, el Juez de Juicio providenciará las pruebas, admitiendo las que sean legales y procedentes y desechando las que aparezcan manifiestamente ilegales o impertinentes. En el mismo auto, e Juez ordenará que se omita toda declaración o prueba sobre aquellos hechos en que aparezcan claramente convenidas las partes. (p. 65).

\section{La promoción de la prueba}

Las prevenciones que despertaba el documento electrónico desde la óptica probatoria civil, se originaban a raíz de la comparación de las características propias del documento en soporte papel y aquellas que presentaba en sus inicios el contenido en soporte informático.

Por remisión de la LMDFE al sistema de promoción de pruebas libres consagrado en el CPC y por virtud del principio de equivalencia funcional, los mensajes de datos deben promoverse, en principio, bajo la prueba documental. No obstante, al tratarse de un procedimiento que emplea la técnica en la generación del soporte documental, es necesario el auxilio de una experticia y/o inspección judicial según el caso.

La Ley de Servicios de la Sociedad de la Información y del Comercio Electrónico Española (LSSICE) (2002) en su artículo 24.2, establece que "el soporte electrónico en que conste un contrato celebrado por vía electrónica, será admisible en juicio como prueba documental". (p. 10). En la LMDFE no se encuentra una disposición que establezca, en forma expresa que los mensajes de datos deben promoverse bajo la forma documental. Sin embargo, en virtud del principio de equivalencia funcional que rige las operaciones de comercio electrónico y por remisión al CPC, donde se establece la aplicación analógica en el tratamiento de las pruebas libres, la vía de incorporación adecuada es la prueba documental.

Al tratarse de una prueba documental de naturaleza electrónica, debe aportarse no mediante forma impresa, sino en su soporte informático: el disquete, el CD ROM, el disco duro del computador o mediante su envió telemático a través de Internet o cualquier otra red que permita la intercomunicación. Para cumplir con los lapsos procesales, es recomendable solicitar el apoyo de un prestador de servicios de certificación a objeto de determinar el momento de la remisión del documento al tribunal, a través del sistema de sellado de tiempo.

En la legislación venezolana y más concretamente en derecho del trabajo la promoción de documentos electrónicos debe realizarse en la audiencia preliminar primigenia como lo establece el artículo $73 \mathrm{de}$ la Ley Orgánica Procesal del Trabajo (2002) que señala: "La oportunidad de promover pruebas para ambas partes será en la audiencia preliminar no pudiendo promover pruebas en otra oportunidad posterior salvo las excepciones establecidas en esta Ley". (p. 64)

\section{Valoración pertinente del medio probatorio acorde con el ordenamiento jurídico venezolano}

La interpretación para la apreciación y posterior valoración de la prueba judicial, constituye un derecho del ciudadano previsto en la Constitución de la República Bolivariana de Venezuela (1999) (p. 41) en su artículo 49.

Pues la actividad probatoria en el proceso, involucra el derecho a preservar las pruebas, proponerlas en el proceso, oponerse a su admisión, a que sean admitidas y ordenadas, a que sean evacuadas $y$ 
controlarlas y por último, a que sean valoradas por el operador de justicia".

Por valoración o apreciación de la prueba judicial, como expresa Devis-Echandía, (1981), se entiende "la operación mental que tiene por fin conocer el método o valor de convicción que pueda deducirse de su contenido" (p. 267). Luego el acto de apreciación o valoración de la prueba, es un acto exclusivo del operador de justicia, que debe ser realizada al momento final del proceso, esto es justo al emitir el fallo definitivo, pues es en ese momento que debe analizar todo el caudal probatorio cursante en autos.

La fijación de los hechos se define como la estabilización de los mismos en la sentencia, como consecuencia de su demostración a través de los medios probatorios interpretados, valorados y apreciados por el juez, es decir, afirmado el hecho y demostrado en el proceso, una vez que el mismo ha sido reconstruido o historificado, debe tenerse presente en la sentencia para que sirva de supuesto concreto de la norma jurídica contentiva de la consecuencia legal pretendida por las partes.

La valoración de la prueba judicial será el acto posterior a su interpretación o análisis concreto y aislado, tendiente a atribuir a cada prueba su grado de convicción o certeza, más aún credibilidad demostrativa de hechos controvertidos.

El español Montero, (1998), expresa: ...el conjunto de interpretaciones y valoraciones que realice el juez, puede denominársele apreciación de la prueba, que consiste en operaciones mentales que ha de realizar el juzgador, para que partiendo de los medios probatorios aportados en el proceso, llegar a establecer la certeza respecto a las afirmaciones que se refieren al supuesto fáctico de la norma contentiva de la consecuencia jurídica solicitada. (p. 269)

Ahora bien en el caso concreto de la investigación, una vez incorporado el documento electrónico al proceso, entran en juego los distintos sistemas de valoración de la prueba. El principal inconveniente que se observa está directamente relacionado por los parámetros a los cuales debe someterse el juzgador en el momento de su valoración, esto es, si debe valorar los documentos electrónicos atendiendo a las reglas de la sana critica o si por el contrario, existe un sistema legal de valoración en concreto y el juez no puede apartarse de él.

En relación al sistema de valoración de la prueba acogido por el legislador venezolano en el marco de la Ley Orgánica Procesal del Trabajo, el mismo fue el de libre convicción razonada o sana critica, tal como lo expresa el artículo 10 de la Ley Orgánica Procesal del Trabajo (2002) al señalar: "Los Jueces del trabajo apreciaran las pruebas según las reglas de la sana critica; en caso de duda, preferirán la valoración más favorable al trabajador". (p. 42)

El citado artículo guarda concordancia con lo establecido en el artículo 507 del CPC que textualmente reza: "A menos que exista una regla legal expresa para valorar el mérito de la prueba, el Juez deberá apreciarla según las reglas de la sana critica". (p. 99)

\section{CONCLUSIONES}

En el derecho laboral venezolano la promoción de medios de pruebas electrónicos como documentos debe realizarse en la audiencia preliminar primigenia como lo establece el artículo 73 de la Ley Orgánica Procesal del Trabajo.

Los medios de prueba electrónicos por remisión expresa de la Ley Sobre Mensajes Datos y Firmas Electrónicas, deben aportarse al proceso judicial, conforme al sistema de promoción de pruebas libres consagrado en el Código de Procedimiento Civil y por virtud del principio de equivalencia funcional, los mensajes de datos deben promoverse, en principio, bajo la prueba documental. No obstante, al tratarse de un procedimiento que emplea la técnica en la generación del soporte 
documental, es necesario el auxilio de una experticia y/o inspección judicial según el caso.

Después del breve análisis efectuado pueden obtenerse algunas conclusiones en lo que se refiere a la determinación de las características esenciales del documento electrónico o, más bien dicho, informático. Estas características esenciales serían las siguientes: 1. El documento debe estar escrito en lenguaje binario. 2. El documento debe estar o poder ser almacenado en soporte informático, magnético, óptico o cualquier otra clase de soporte que pudiera ser desarrollado para tales fines. 3. El documento debe poder ser, mediante la aplicación del correspondiente programa informático,

La diferencia entre un documento electrónico y un documento en soporte de papel, o si el documento electrónico es técnicamente un documento, encuentra su mayor justificación en el ámbito relativo al campo procesal civil pues, en tal contexto el documento ha sido entendido circunscrito al escrito en soporte papel, circunstancia que también se ha dado en la esfera relativa al derecho civil sustantivo, en donde, específicamente en el ámbito propio de la realidad negociar-, el documento se ha vinculado en forma precisa a la manifestación de voluntades con finalidad negociar.

\section{REFERENCIAS}

Arias, F. (2006). El Proyecto de Investigación. (5ta edición). Editorial Episteme. (p.27)

Barriuso Ruiz, C. (1998). La contratación electrónica., Editorial Dykinson, Madrid, España

Carnelutti, F. (1982). La Prueba Civil. Tr: Santiago Sentis Melendo. Editorial Jurídica Europa América, Buenos Aires, Argentina (p 450)

Código de Procedimiento Civil. Gaceta Oficial № 4.209 Extraordinaria de fecha (1990 septiembre 18)
Constitución de la República Bolivariana de Venezuela. Gaceta Oficial de la República Bolivariana de Venezuela №- 36860. (1999 diciembre 30). Caracas Venezuela.

Davara R., M. (1998). La Protección de datos en Europa, Universidad Pontificia de Comillas, Madrid, (p. 97)

Devis-Echevería, H., (1981). Teoría general de la prueba judicial. Tomo. I, 5 Ed. Victor D. de Zabalía, Editor Buenos Aires. Argentina

Jijena, R. (1998). Naturaleza Jurídica y Valor Probatorio del Documento Electrónico, La Ley, número 4.586, (p.1457)

Ley Especial Contra Los Delitos Informáticos. Gaceta Oficial $\mathrm{N}^{\circ} 37.313$ de fecha (2001 octubre 30)

Ley Orgánica Procesal del Trabajo. Gaceta Oficial $\mathrm{N}^{\circ} 37.504$ de fecha (2002 agosto 13)

Ley Sobre Mensajes de Datos y Firmas Electrónicas. Gaceta Oficial No 37.148 de fecha (2001 febrero 28)

Ley de Enjuiciamiento Civil. 1/2000, de 7 de enero, Publicado en: "BOE" núm.7, de 08/01/2000. Referencia: BOE-A-2000323.

https://www.boe.es/buscar/pdf/2000/B OE-A-2000-323-consolidado.pdf

(Consultado: 3/1/2019)

Ley de Servicios de la Sociedad de la Información y de Comercio Electrónico (LSSI). (2002 de 11 de julio) Publicado en: "BOE" núm. 166, de 12/07/2002.Referencia: BOE-A-200213758. https://www.boe.es/buscar/act.php?id=B OE-A-2002-13758. Consultado: 3/1/2019

Montero A., J. (1998) La Prueba en el Proceso Civil. Editorial Civitas. Madrid, España

Rico C., M. (2005) Comercio Electrónico Internet y Derecho. 2da Edición Ediciones. Legis. S.A Colombia

Tamayo y Tamayo, M. (1999). El Proceso de la Investigación Científica. Limusa Noriega Editores. México

Tamayo y Tamayo, M. (2005). El Proceso de la Investigación Científica. Limusa Noriega Editores. México. 